\title{
BMJ Open The effect on quality of life of vitamin $D$ administration for advanced cancer treatment (VIDAFACT study): protocol of a randomised controlled trial
}

\author{
Montserrat Martinez-Alonso, ${ }^{1,2}$ Adriana Dusso, ${ }^{3}$ Gemma Ariza, ${ }^{4}$ Maria $\mathrm{Nabal}^{5}$
}

To cite: Martinez-Alonso M, Dusso A, Ariza G, et al. The effect on quality of life of vitamin $D$ administration for advanced cancer treatment (VIDAFACT study): protocol of a randomised controlled trial. BMJ Open 2014;4: e006128. doi:10.1136/ bmjopen-2014-006128

- Prepublication history for this paper is available online. To view these files please visit the journal online (http://dx.doi.org/10.1136/ bmjopen-2014-006128).

Received 16 July 2014 Revised 12 November 2014 Accepted 14 November 2014

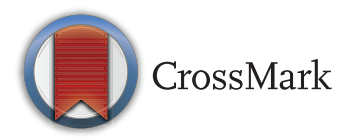

${ }^{1}$ Ciències Mèdiques Bàsiques, Universitat de Lleida, Lleida, Spain ${ }^{2}$ Biostatistics and Methodological Support, IRBLLEIDA, Lleida, Spain ${ }^{3}$ Experimental Nephrology, IRBLLEIDA, Lleida, Spain

${ }^{4}$ Rehabilitation Unit, Hospital Universitari Arnau de Vilanova, Lleida, Spain ${ }^{5}$ Palliative Care Supportive Team, Hospital Universitari Arnau de Vilanova, Lleida, Spain

\section{Correspondence to} Montserrat Martinez-Alonso; montserrat.martinez@cmb. udl.cat

\section{ABSTRACT}

Introduction: Vitamin $D$ is related to resistance to chronic diseases, physiological parameters and functional measures. All of these relationships underscore the potential benefits of cholecalciferol or D3 (nutritional vitamin D) in cancer. This is the first study designed to obtain conclusive evidence on the effect of cholecalciferol in advanced patients with cancer. The main goal is to assess its effects on the patient's perceived quality of life. Cholecalciferol's impact on fatigue and physical performance, as well as its cost utility, will also be assessed.

Methods and analysis: A randomised triple-blind phase II/III placebo-controlled multicentre trial has been designed. Patients satisfying the inclusion and exclusion criteria will be randomly assigned to receive cholecalciferol or placebo. Eligible patients will be adults with a locally advanced or metastatic or inoperable solid cancer in palliative care, who have given signed informed consent and have matched inclusion and exclusion criteria. The randomisation will be based on a computer-generated procedure and centralised by the pharmacy service of the coordinating centre. The assigned treatment will be administered by the hospital's pharmacy to conceal group allocation for patients and healthcare providers. Cholecalciferol (4000 IU/day) or placebo, starting at day 15 and continuing up to day 42 , will be added to palliative care treatment. Outpatient visits will be scheduled every 14 days.

Ethics and dissemination: Ethical approval was received from the Medical Ethical Commitee of the HUAV (CEIC-1169). Participants and their families will receive the research findings which will also be disseminated on local and national media, presented at national and international meetings of the specialty, and published in peer-reviewed scientific journals.

Trial registration number: EudraCT: 2013-003478-29.

\section{INTRODUCTION}

\section{Background and rationale}

Cancer-related fatigue is the most common symptom in patients in palliative care with an overall prevalence of $90 \%$ at terminal stages. ${ }^{1}$ However, cancer-related fatigue cannot be

\section{Strengths and limitations of this study}

- At least a clinically relevant improvement in the quality of life is expected for oncological patients with no possibility of curable treatments.

- It is a multidisciplinary approach to patient care which includes coordination with physiatrists in functional rehabilitation.

- Cost utility analysis of vitamin D supplementation will allow informed decision-making.

- Potential limitations are that the amount of sun exposure, although expected to be low for patients with advanced cancer in palliative care, has not been taken into account.

easily diagnosed, since it may be masked by pain, dyspnoea or nausea. ${ }^{2}$ It often becomes apparent once it compromises basic daily life activities. ${ }^{3}$ Additionally, there are also subjective, psychological and emotional components of the impact of lack of strength on each individual, which can be appropriately assessed by currently available questionnaires which measure the patient's perception of the impact of fatigue on his/her quality of life. $^{45}$

The pathogenesis of cancer-related fatigue is multicausal and involves physiological and biochemical disorders directly associated with tumour progression, severe adverse effects resulting from cancer treatment, as well as physical and psychological stress, anaemia, lack of exercise, chronic pain and severe uncontrolled symptoms. ${ }^{6} 7$

At present, a variety of interventions have been studied with reportedly small but significant benefits such as drug therapy, ${ }^{8-10}$ which are not free of adverse effects. Psychosocial and physical interventions on fatigue intensity are also available for patients with cancer. ${ }^{11}$ However, in patients in palliative care, recommendations for therapy are scarce. Functional rehabilitation ${ }^{12}$ should be 
tailored to each patient to help maintain a relatively active daily life. The goal is not to recover a specific level of activity, but to maintain physical endurance so that rest or inactivity will not reduce muscle reserve.

Vitamin D is a fat-soluble vitamin present in only a few foods, but which can be obtained from sun exposure and supplements in its biologically inert form. It must undergo two hydroxylations in the body for activation. First, the liver converts vitamin $\mathrm{D}$ to 25-hydroxyvitamin D $(25(\mathrm{OH}) \mathrm{D})$, and afterwards the physiologically active 1.25-dihydroxyvitamin $\mathrm{D}(1.25(\mathrm{OH}) 2 \mathrm{D})$ is produced mainly by the kidneys.

A recently published review ${ }^{13}$ supports numerous reports delineating the multiple mechanisms underlying vitamin D prosurvival actions experimented with at the laboratory. Their findings show that vitamin $\mathrm{D}$ deficiency results in a defective control of: (1) Cell growth causing hyperproliferative disorders, genomic instability, Tumour necrosis factor Alpha Converting Enzyme (TACE)-driven metastasis, exacerbated growth and immune escape; and accelerated progression of cancer lesions; ${ }^{14-17}$ (2) DNA repair mechanisms causing age-associated disorders and resistance to therapy in cancer; ${ }^{16}{ }^{18}$ (3) Muscular weakness and impaired neuromuscular function; ${ }^{19}{ }^{20}$ (4) Musculoskeletal pain derived from cancer treatment; ${ }^{21}$ (5) The immune system causing increased antigenicity in antigen presenting cells, reduced content of T-regulatory lymphocytes, systemic inflammation and multiple organ damage, as well as a higher propensity for autoimmune disorders; ${ }^{15} 19$ (6) The integrity of the FGF23/klotho responsible for the antiageing properties of klotho in the kidneys, in protecting the vasculature from atheromatosis and calcification, and as a tumour suppressor and protector from the onset of resistance in cancer treatment; ${ }^{22-24}$ (7) The renin-angiotensin system to prevent hypertension, renal and cardiovascular damage due to excessive oxidative stress; ${ }^{25}{ }^{26}$ (8) Acquisition of atherothrombotic phenotype of circulating monocytesmacrophages and increased severity of atherosclerotic lesions; ${ }^{27}$ (9) Podocyte function to protect from proteinuria and proteinuria-induced cardiovascular lesions. ${ }^{28} 29$

When no curable treatment is possible, palliative care is directed towards minimising symptoms, relieving suffering and bringing patients to the best possible health-related quality of life (HRQoL). Avoiding the toxicity associated with some palliative treatments is a must in these patients. There is an urgent need to develop new interventions in this population based on current evidences, namely: (1) The strong epidemiological association between vitamin $\mathrm{D}$ deficiency and the high risk of mortality from all causes in the general population ${ }^{15}$ and specifically from cancer; ${ }^{30} 31$ (2) The high frequency of vitamin $\mathrm{D}$ deficiency in patients with cancer, estimated to be between $47 \%^{32}$ and $88 \% ;^{33}$ (3) The anticancer, antiageing, antipain, immunomodulatory and the protective renal, cardiovascular and neuromuscular properties of a normal vitamin D status; ${ }^{15} 193435$ and (4) The significant $11 \%$ reduction in all cause mortality estimated for D3 by combining all randomised controlled trials. ${ }^{30}$ We present a clinical trial designed to obtain, for the first time, evidence-based recommendations on the efficacy of vitamin D3 supplementation, a safe, non-costly therapeutic strategy, in improving physical performance, decreasing fatigue and increasing HRQoL in patients with advanced cancer in palliative care.

\section{OBJECTIVES}

The primary objective of this study is to evaluate the efficacy of the administration of vitamin D3 to enhance patient-reported HRQoL.

The secondary objectives of this study are: (1) To evaluate the efficacy of the administration of vitamin D3 for enhancing physical performance, decreasing perceived fatigue and achieving serum levels of $25(\mathrm{OH}) \mathrm{D}$ above $30 \mathrm{ng} / \mathrm{mL}$; (2) To evaluate the effect on tumour biomarkers; (3) To explore the relationship between vitamin D treatment compliance and $25(\mathrm{OH}) \mathrm{D}$ levels; (4) To explore the relationship between $25(\mathrm{OH}) \mathrm{D}$ levels and renal function; (5) To explore the dose-response relationship in the group of patients with vitamin D3 for the main outcome; (6) and to assess the cost utility of the proposed administration of vitamin D3.

\section{METHODS AND ANALYSIS}

The tolerable upper intake level $(\mathrm{UL}=4000 \mathrm{IU} /$ day or $100 \mu \mathrm{g} /$ day) has already been established by the European Food Safety Authority for the adult population. ${ }^{36}$ However, there is a need for a proof of concept trial to be conducted in patients with advanced cancer in palliative care to gain preliminary data on the safety and efficacy of high doses (at UL) of vitamin D. Therefore, a phase II proof of concept study is planned to obtain reliable data to support a phase III confirmatory trial, within a randomised controlled trial with a placebo two-stage adaptive design.

\section{Trial design}

A randomised triple-blind phase II/III placebo-controlled multicentre trial has been designed. The randomisation will be based on a computer-generated procedure centralised by the pharmacy service of the coordinating hospital in order to ensure the required treatment allocation concealment. Serum levels of vitamin $\mathrm{D}$ will be not available for the patients or their physicians to preserve treatment allocation concealment. Randomisation will be stratified by primary solid tumour type in a 1:1 ratio. In a proof of concept trial (phase II or stage 1), patients will be assessed after the first 14 days of treatment about selfperceived changes in HRQoL. The study includes a first interim analysis similar to a futility test to discontinue the trial, and reject vitamin D3, if no better outcome is obtained when compared to the placebo group. Otherwise, it will continue to Phase III (stage 2), which is planned to establish the efficacy in improving and 
maintaining HRQoL as well as the safety of the treatment with UL of vitamin D over placebo for 28 days (till day 42 from enrolment). This study will assess the efficacy of vitamin $\mathrm{D}$ when administered in combination with the usual palliative care and functional rehabilitation.

\section{Study setting}

Phase II of the study will take place at Hospital Universitari Arnau de Vilanova (Lleida, Spain). For phase III of the study, three additional Spanish hospitals, Duran $i$ Reynalds (Barcelona), La Paz (Madrid) and Virgen de la Macarena (Sevilla), will participate.

\section{Patients}

Eligible patients will be adults with a locally advanced or metastatic or inoperable solid cancer in palliative care on signed informed consent. Exclusion criteria will be having a Karnofsky $<30 \%$, being pregnant or breastfeeding females, undergoing severe liver or renal (glomerular filtration rate $<60 \mathrm{ml} / \mathrm{min}$ ) failure, having a cognitive deterioration (more than 5 mistakes in the Pfeiffer test), suffering from significant pain, dyspnoea, nausea or vomiting (more than 6 out of 10 in the corresponding Numerical Rating Scale 0:10), hypercalcaemia (> $10.5 \mathrm{mg} / \mathrm{dL}$ ), having received chemotherapy or radiation therapy within the past 3 weeks prior to inclusion, or having the possibility of initiating a new cycle of chemotherapy or radiation therapy within a period of 6 weeks after their inclusion date.

A prescreening day is scheduled for each adult patient with a locally advanced or metastatic or inoperable solid cancer in palliative care who did not present any known reason to be excluded. On that day, a blood test will be ordered (since results should be available on the screening day for the complete assessment of exclusion criteria) and a visit with the physical rehabilition medical doctor will be scheduled for the same day.

On the screening day, those patients satisfying inclusion and exclusion criteria will be provided with complete information about the study and enrolled into the study pon signed informed consent.

\section{Intervention}

Vitamin D3 supplements of $4000 \mathrm{IU} /$ day (UL) or placebo will be added to palliative care treatment on day 14 after enrolment and continued up to day 42. Outpatient visits will be scheduled every 14 days. Supportive evidence for the UL established $\operatorname{dose}^{36}$ is provided by randomised controlled studies in which this quantity or higher was administered to various population groups for up to 12 months without evidence of persistent hypercalcaemia or hypercalciuria.

Palliative care treatment will be standardised among participating physicians to ensure comparable procedures on symptoms control (pain, nausea, vomiting, constipation, diarrhoea, lack of appetite, shortness of breath, cough and dry mouth).
Functional rehabilitation will be coordinated by the rehabilitation doctor at each participating hospital from day 0 . On that day, there will be an evaluation of the clinical and functional status (weakness, effort tolerance, mobility, functional performance and independence in activities of daily living), neuro-orthopaedic disorders, orthopaedic aids necessary to improve the functionality and individualised physical therapy to perform. Three additional sessions will be scheduled before day 14 to work in the following basic protocols: Assisted and free active physical therapy; Respiratory physiotherapy; Rehabilitation of trunk balance in sitting and standing; Re-education in walking; Energy saving measures, and teaching family involvement. Any requirement for additional measures of rehabilitation will be specifically noted. Leaflets containing advice and recommended exercises for functional rehabilitation will be provided to all participating patients and their families.

\section{OUTCOMES}

\section{Primary outcome}

Change in patient self-reported HRQoL is the primary outcome. It will be assessed using the European Organisation for Research and Treatment of Cancer (EORTC) QLQ-C15-PAL, ${ }^{37}$ a questionnaire developed for patients with cancer in palliative care. It includes rates on: pain, physical function, emotional function, fatigue, global health status/quality of life, nausea/ vomiting, appetite, dyspnoea, constipation and sleep. Its scale rating global health status/quality of life using a numbered scoring scale from 1 or very poor to 7 or Excellent is the primary outcome. An anchor question approach is included in order to establish ranges of scores reflecting patient self-assessment of changes in perceived HRQoL (as an approach to minimal important difference assessment).

Since patients with advanced cancer in palliative care can undergo rapid health deterioration (within a few days), primary as well as secondary outcomes will be recorded at baseline and on the allocation day (14 days after enrolment) in order to assess intrapatient variability occurring before any intervention. After starting the intervention, primary and secondary outcomes will be collected on days 28 and 42 from enrolment day (after 14 days and 28 days of taking vitamin $\mathrm{D}$ or placebo).

\section{Secondary outcomes}

Change in cancer-related fatigue is the second most important outcome, and will be assessed with the fatigue subscale of the Functional Assesment of Cancer Therapy:Fatigue (FACT-F) questionnare. ${ }^{38}$ FACT-F is a widely used 13-item fatigue subscale where each item is a five-point Likert self-report scale ranging from $0=$ 'not at all' to $4=$ 'very much so'. The total score varies from $0=$ 'worst condition' to $52=$ "best condition'.

Change in functional capacity is another important secondary outcome and will be assessed using the 
Barthel Scale, ${ }^{39}$ an ordinal scale that measures performance in activities of daily living, as well as the Karnofsky and the Palliative Performance Scale (PPS) scores, ${ }^{40} 41$ which are used to quantify patients' performance status. All of them provide a score from 100 to 0 , where 100 indicates no evidence of disease (normal performance) and 0 indicates death.

Other secondary outcomes include changes in serum levels of 25-hydroxyvitamin $\mathrm{D}$, changes in tumoural biomarkers, adherence to the randomised intervention (defined by the proportion of compliance with the randomised intervention out of the total 28 days), renal function and cost utility analyses. Utilities will be based on the patient self-reported HRQoL measured by EuroQoL (EQ-5D; $3 \mathrm{~L}$ ). This questionnaire assesses five dimensions (mobility, self-care, usual activities, pain/discomfort, anxiety/depression) to define the health state of patients. Each health state will be translated in the corresponding valuation by applying the Spanish general population utility weights, resulting in valuations from 1 (optimal health) through 0 (as bad as death) to negative values (worse than death). Direct costs will be collected in both groups.

\section{Sample size}

The research team considers that a $15 \%$ difference is clinically relevant and that differences below $15 \%$ will not suffice to demonstrate the clinical significance of the effect of supplementation with vitamin D on quality of life. Therefore, this study is designed to detect differences of $15 \%$ or more in the proportion of patients with improved HRQoL between the groups. Error probabilities of $\alpha$ and $\beta$ to 0.05 and 0.2 , respectively, for a unilateral contrast with a 1:1 allocation are fixed for sample size calculations.

The phase II sample size is designed to control early stopping probability based on the exact binomial distribution (Simon, 1989) on optimal two-stage designs and fixed to at least 28 patients per group (optimal design for the first stage). The trial will be stopped if the number of patients that improve is not higher in the intervention group than in the control group.

The total sample size for this study (adding phases II and III) is computed as the minimal number of patients per group to detect differences from $15 \%$, even in the case of maximal requirements that would be having proportions of improvement in HRQoL close to $50 \%$. Therefore, with proportions of improvement in HRQoL of $42.5 \%$ for the placebo group and $57.5 \%$ for the vitamin $\mathrm{D}$ group, and using the same error probabilities in a unilateral contrast, a minimum of 137 patients per group (a total of 274 patients enrolled) are estimated as the minimum sample size required by applying the arcsinus approximation. Since twice this improvement could be observed in the vitamin $\mathrm{D}$ group, an interim analysis is scheduled at the end of treatment for the first 43 patients per group (phase II patients included) in order to minimise the number of patients in the placebo group. If the difference in the proportion of patients with minimally improved HRQoL is twice the expected $15 \%$ in favour of vitamin D3, the trial will be stopped for ethical reasons for the benefit of the patients in the placebo group. In other words, the second interim analysis is planned with a stopping rule so that if the improvement difference is $30 \%$ or greater, all the participants will benefit. Figure 1 summarises this study design.

\section{Assignment of interventions}

The randomisation process will be centralised at the Pharmacy Service of the Hospital Universitari Arnau de Vinalova. The Pharmacy Service will be the responsible for randomising and blindly assigning vitamin D3 or placebo to each patient, stratified by the primary tumour and blocking it to ensure the same number of patients in each intervention. Drug or placebo formulation, labelling and allocation will be performed by the pharmacist, who will create an identifier for each patient, independently from the order of entry into the study. The matching of each patient identifier with its allocated intervention will be saved in an independent database for the exclusive use of the pharmacist or the External Safety Monitoring Committee until the end of the study.

\section{Data collection and management}

Figure 2 summarises the data collection and management description that is detailed below.

\section{Data collected exclusively at baseline (day $\mathbf{0}$ )}

The inclusion and exclusion criteria checklist and baseline information will be also collected including sociodemographical data (age, sex, education), anthropometric data (height), clinical data (primary tumour, stage and treatment), as well as treatments that may interact with vitamin D (antiepileptics, antihypercholesterolaemic, certain diet drugs and corticosteroids).

\section{Data collected at all visits (days $0,14,28$ and 42)}

Collection will include: anthropometric data (weight, body mass index, triceps skinfold); palliative care treatments (cachexia, pain,...); functional capacity (Barthel, Karnofsky, PPS); cancer-related fatigue (FACT-F

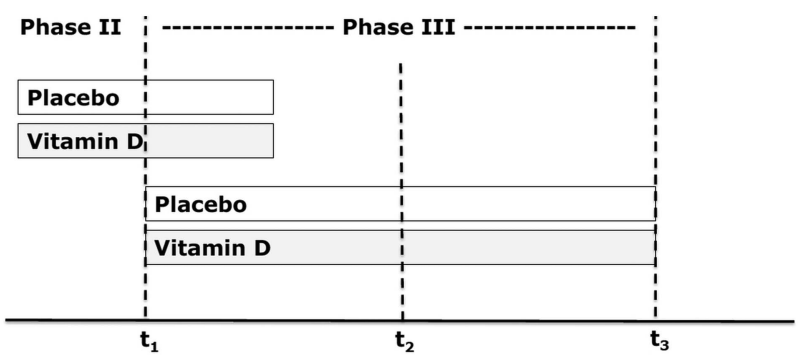

$\mathbf{t}_{\mathbf{1}}$ : Interim analysis, after 28 patients per arm were assessed till day 28 $\mathbf{t}_{2}$ : Interim analysis, after 43 patients per arm were assessed till day 42 $\mathbf{t}_{3}$ : Final analysis, after 137 patients per arm were assessed till day 42 .

Figure 1 Trial design. 
Figure 2 VIDAFACT participant timeline.

\begin{tabular}{|c|c|c|c|c|c|}
\hline & \multirow[t]{2}{*}{\begin{tabular}{|c|} 
Pre- \\
screening*
\end{tabular}} & \multicolumn{2}{|c|}{ Pre-intervention } & \multicolumn{2}{|c|}{$\begin{array}{c}\text { Intervention } \\
\text { (vit. D or placebo) }\end{array}$} \\
\hline & & $\begin{array}{c}\text { Day 0 } \\
\text { (Enrolment) }\end{array}$ & $\begin{array}{c}\text { Day 14 } \\
\text { (Allocation) }\end{array}$ & Day 28 & Day 42 \\
\hline Assessment of available Inclusion and Exclusion criteria & $\bullet$ & & & & \\
\hline Blood test order & $\bullet$ & & $\bullet$ & $\bullet$ & $\bullet$ \\
\hline Complete assessment of inclusion and Exclusion criteria & & $\bullet$ & & & \\
\hline Onset of the intervention in physical rehabilitation & & $\bullet$ & & & \\
\hline Socio-demographical data (age, sex, education) & & $\bullet$ & & & \\
\hline Antropometrical data (height) & & $\bullet$ & & & \\
\hline Clinical data (primary tumour, stage and treatment) & & $\bullet$ & & & \\
\hline Treatments that could interact with vitamin D & & $\bullet$ & & & \\
\hline $\begin{array}{l}\text { Anthropometric data } \\
\text { (weight, BMI, tricipital skinfold thickness) }\end{array}$ & & $\bullet$ & $\bullet$ & $\bullet$ & $\bullet$ \\
\hline Paliative care treatments & & $\bullet$ & $\bullet$ & $\bullet$ & $\bullet$ \\
\hline Biochemical data & & $\bullet$ & $\bullet$ & $\bullet$ & $\bullet$ \\
\hline Functional capacity scales (Barthel, Karnofsky, PPS) & & $\bullet$ & $\bullet$ & $\bullet$ & $\bullet$ \\
\hline Cancer-related fatigue (FACT-F subscale) & & $\bullet$ & $\bullet$ & $\bullet$ & $\bullet$ \\
\hline $\begin{array}{l}\text { Health related quality of life } \\
\text { (EORTC QLQ-C15-PAL, EQ5D) }\end{array}$ & & $\bullet$ & $\bullet$ & $\bullet$ & $\bullet$ \\
\hline Patient perceived change in health related quality of life & & & & $\bullet$ & $\bullet$ \\
\hline Adherence to the randomized intervention & & & & $\bullet$ & $\bullet$ \\
\hline Safety variables & & & & $\bullet$ & $\bullet$ \\
\hline Adverse events & & & & $\bullet$ & $\bullet$ \\
\hline
\end{tabular}

* From 1 to 7 days previous to day 0 (enrolment). subscale); and quality of life (EORTC QLQ C15 PAL and EQ-5D). Biochemical data will be separated into one part available during the study for the patient and the physician (PCR, total protein, serum albumin, triglycerides and cholesterol (total, low-density lipoprotein, high-density lipoprotein)) and another available during the study only for the external Data Monitoring Committee (25-hydroxyvitamin D, serum calcium and urinary calcium).

\section{Data collected in the postallocation visits (days 28 and 42)}

Patient assessment of change from the start of treatment will be collected in response to the question: Please indicate whether there has been any change in your health related quality of life from the start of treatment by choosing one of the following options: -3 , very much worse; -2 , much worse; -1 , slightly worse; 0 , no change; 1 , a little better; 2 , much better; 3 , very much better. An at least minimal improvement is defined for patients with an answer from 1 to 3 to this question.

Reported compliance per day starting on day 15 will be also collected.

Most people are not expected to suffer from any side effect, unless they take more than the prescribed amount of vitamin D. Hypercalcaemia and patient assessment of weakness, fatigue, drowsiness, headache, loss of appetite, dry mouth, metallic taste, nausea or vomiting are the adverse events listed in the bibliography. Adverse events will be collected using an ordinal scale of four categories for each one: 'No ( 0 points)', 'Mild (1 points)', 'Moderate (2 points)' and 'Severe (3 points)'. All adverse events happening postintervention (from 15th to 48th day) will be recorded throughout the study, registering: the adverse event name, starting and ending dates, frequency, severity, intensity, relationship to study drug, action taken and resolution.

Any suspected unexpected serious adverse reaction (SUSAR) will be reported to the Spanish Agency
(AEMPS). An SUSAR is defined as any adverse reaction to vitamin $\mathrm{D}$ which shows a reasonably and unexpected causal relationship, whose nature, severity or outcome is not consistent with the summary of the characteristics of the technical data and that produces death, endangers the patient's life immediately, produces a persistent or significant disability, requires or prolongs hospitalisation, or becomes a significant danger that is comparable to the above criteria.

\section{Statistical analysis}

For the first interim analysis, only the number of patients with at least minimally improved HRQoL will be compared, with no statistical test to base the decision to stop. For the second interim analysis, a difference between response rates of $30 \%$ or higher will be used to make the decision to stop. At the end of the study, a descriptive univariate analysis of each group will be performed, followed by a comparison between groups to check if randomisation produced comparable groups in each potential confounder. Bivariate analyses will use the Mann-Whitney or $\chi^{2}$ test for quantitative or qualitative variables, respectively. In case of differences, analysis of minimally improved HRQoL by logistic regression will be performed adjusting for them. For differences between groups in longitudinal measurements (such as chemistries), linear and non-linear mixed-effect models will be used. Additionally, for the intervention group (patients treated with vitamin $\mathrm{D}$ ), longitudinal measures of serum vitamin $\mathrm{D}$ and renal function will be related by fitting mixed effects linear models. Main outcomes will be analysed by intention to treat. A longitudinal analysis of treatment compliance (cumulative dose in both periods of 14 days) will be performed in relation to serum vitamin $\mathrm{D}$ levels as well as an analysis per protocol to look at dose-response effects. Finally, a cost utility analysis of the intervention with cholecalciferol will be performed. For each patient, quality-adjusted life years 
will be calculated as the area under the EQ-5D utility curve. Since patients with missing values from questionnaires are likely to be worse than those with the available measurements, the missing outcomes will be imputed using non-linear regression models. A $\mathrm{p}<0.05$ will be established as statistically significant. R software will be used. $^{42}$

\section{ETHICS AND DISSEMINATION}

Any amendment will be immediately communicated to both agencies. All participants will provide signed informed consent and their confidentiality will be guaranteed by encoding all personal information. Insurance will cover any unexpected damage attributable to participation in the study.

\section{Ethical implications}

An external Safety Monitoring Committee has been created, comprised of one physician external to the study from each of the participating hospitals. They will be responsible for monitoring participants' calcium and vitamin D levels during the study in order to ensure the patients' safety and allocation concealment. The research team has no economical interest in favour of or against vitamin D supplementation. Its only interest is to provide evidence as to whether vitamin D supplementation is beneficial or not for patients with advanced cancer in palliative care. Criteria to stop after any of the two planned interim analyses are clearly stablished in the study protocol. After each interim analysis, blindly performed by the statistician of the research team, the pharmacy service will assess the results of interim analyses in relation to the stopping rules established in the protocol and with their exclusive knowledge of the intervention groups. They will be responsible for communicating the end or the continuation of the study based on the criteria established in the study protocol to the research team. The two interim analyses and the phase II/III study design are part of this protocol to minimise the use of time and patients required to demonstrate whether, as hypothesised, there is a clinically significant benefit from the use of vitamin $\mathrm{D}$ in patients with advanced cancer in palliative care. The size of the group randomly assigned to placebo is minimised during the study and, if the effect of vitamin D interventions become conclusive, this group will also be supplemented with vitamin D.

\section{Dissemination policy}

Given this project's scientific and social interest, its results will be locally and internationally disseminated in congresses, scientific papers and the media.

\section{DISCUSSION}

The potentially high scientific and social interests of this project, fully based on the premise that 'When no curable treatment is possible, palliative care should be directed towards minimizing symptoms, relieving suffering and bringing patient to the best possible health related quality of life', can be summarised as follows:

Scientifically:

1. The results of this safe, cost-effective intervention with vitamin $\mathrm{D}$ supplementation in patients in palliative care research would be, to the best of our knowledge, the first attempt to conclusively address whether the quality of life for these patients could also improve from the prosurvival properties of a normal vitamin D status, as reported not only in healthy individuals but also in those with diabetes, hypertension, cardiovascular disease, autoimmune disorders, tuberculosis, kidney disease and cancer.

2. This work could provide evidence-based recommendations for the efficacy of vitamin D supplementation to patients in palliative care in improving their quality of life. Such a finding could be immediately incorporated into clinical practice due to its low cost and lack of toxicity. Furthermore, if confirmed, these findings should provide a solid basis for the design of prospective, well-powered clinical trials directed at customising vitamin D supplementation regimens in order to expedite the correction of vitamin $\mathrm{D}$ deficiency in treatment-resistant patients and, more importantly, ensure the fast improvement of their quality of life.

3. It could help initiate new lines of research on the pathogenesis of cancer-related fatigue to prevent its onset at early stages and attenuate its progression in cancer.

4. It could bring basic and clinical research together in the arena of advanced cancer.

5. The most optimistic outcome would be to change the 'current paradigm' for patients in palliative care from a state of 'no curable treatment possible' to a vitamin $\mathrm{D}$ healing transition making the patient eligible for a new customised cancer therapy with vitamin $\mathrm{D}$ as a coadjuvant therapy.

Socially:

1. For the individual: The possibility of giving a patient in palliative care a safe, non-costly, and evidencebased effective option to maintain their quality of life, and the hope that they could eventually become eligible to restart cancer therapy for a cure is invaluable.

2. For the community: This is research with a potentially high clinical relevance, completely independent from the interests of the pharmaceutical industry, whose results could benefit patients in palliative care even in underdeveloped countries with the poorest of healthcare systems.

Twitter Follow Montserrat Martinez-Alonso at @pro_minorias

Open Access This is an Open Access article distributed in accordance with the Creative Commons Attribution Non Commercial (CC BY-NC 4.0) license, which permits others to distribute, remix, adapt, build upon this work noncommercially, and license their derivative works on different terms, provided 
the original work is properly cited and the use is non-commercial. See: http:// creativecommons.org/licenses/by-nc/4.0/

Acknowledgements The authors thank Dr Montse Rué at the Basic Medical Sciences Department of the University of Lleida for her valuable suggestions and careful reading of the final version of this manuscript.

Collaborators Josep Porta-Sales; Alberto Alonso; Jaime Boceta.

Contributors MM-A contacted AD, MN and GA to develop the original concept of this study. All authors contributed to discussion and revisions to the study design. MN obtained funding from an organisation with no competing interests. All authors drafted the manuscript, were involved in the revision of the manuscript and agreed on the final approval of the version to be published.

Funding Participants' insurance is covered by a $€ 10000$ donation by Fundación Jesús Serra, from Catalana Occidente.

Competing interests None.

Patient consent Obtained.

Ethics approval Medical Ethical Commitee of the Hospital Universitari Arnau de Vilanova (CEIC-1169).

Provenance and peer review Not commissioned; externally peer reviewed.

Open Access This is an Open Access article distributed in accordance with the Creative Commons Attribution Non Commercial (CC BY-NC 4.0) license, which permits others to distribute, remix, adapt, build upon this work noncommercially, and license their derivative works on different terms, provided the original work is properly cited and the use is non-commercial. See: http:// creativecommons.org/licenses/by-nc/4.0/

\section{REFERENCES}

1. David A, Pelosi A, McDonald E, et al. Tired, weak, or in need of rest: fatigue among general practice attenders. BMJ 1990;301:1199-202.

2. Stone P, Richardson A, Ream E, et al. On behalf of the Cancer Fatigue Forum. Cancer-related fatigue: Inevitable, unimportant and untreatable? Results of a multi-centre patient survey. Ann Oncol 2000;11:971-5

3. Okuyama T, Tanaka $\mathrm{K}$, Akechi $\mathrm{T}$, et al. Fatigue in ambulatory patients with advanced lung cancer: prevalence, correlated factors, and screening. J Pain Symptom Manage 2001;22:554-64.

4. Stewart GD, Skipworth RJ, Fearon KC. Cancer cachexia and fatigue Clin Med 2006;6:140-3.

5. Lane I. Managing cancer-related fatigue in palliative care. Nurs Times 2005;101:38-41.

6. Minton O, Richardson A, Sharpe M, et al. Systematic review and meta-analysis of the pharmacological treatment of cancer-related fatigue. J Natl Cancer Inst 2008;100:1155-66.

7. Barsevick AM, Irwin MR, Hinds $P$, et al. Recommendations for high-priority research on cancer-related fatigue in children and adults. J Natl Cancer Inst 2013;105:1432-40.

8. Lower EE, Fleishman S, Cooper A, et al. Efficacy of dexmethylphenidate for the treatment of fatigue after cancer chemotherapy: a randomized clinical trial. J Pain Symptom Manage 2009;38:650-62.

9. Martin D. The use of psychostimulants in terminally ill patients. Eur J Palliat Care 2001;8:228-32.

10. Kerr CW, Drake J, Milch RA, et al. Effects of methylphenidate on fatigue and depression: a randomized, double-blind, placebo-controlled trial. J Pain Symptom Manage 2012;43:68-77.

11. Davis MP, Khoshknabi D, Yue GH. Management of fatigue in cancer patients. Curr Pain Headache Rep 2006;10:260-9.

12. Chasen MR, Feldstain A, Gravelle D, et al. An interprofessional palliative care oncology rehabilitation program: effects on function and predictors of program completion. Curr Oncol 2013;20:301-9.

13. Feldman D, Krishnan AV, Swami S, et al. The role of vitamin D in reducing cancer risk and progression. Nat Rev Cancer 2014;14:342-57.

14. Bergada L, Pallares J, Maria Vittoria A, et al. Role of local bioactivation of vitamin D by CYP27A1 and CYP2R1 in the contro of cell growth in normal endometrium and endometrial carcinoma. Lab Invest 2014;94:608-22.

15. Adams JS, Hewison M. Update in vitamin D. J Clin Endocrinol Metab 2010;95:471-8.
16. Grotsky D, Gonzalez-Suarez I, Novell A, et al. BRCA1 loss activates cathepsin L-mediated degradation of 53BP1 in breast cancer cells. J Cell Biol 2013;200:187-202.

17. Dusso A, Arcidiacono MV, Yang J, et al. Vitamin D inhibition of TACE and prevention of renal osteodystrophy and cardiovascular mortality. J Steroid Biochem Mol Biol 2010;121:193-8.

18. Gonzalez-Suarez I, Redwood AB, Grotsky DA, et al. A new pathway that regulates 53BP1 stability implicates Cathepsin $L$ and vitamin D in DNA repair. EMBO $\mathrm{J} 2011 ; 30: 3383-96$.

19. Bouillon R, Carmeliet G, Verlinden L, et al. Vitamin D and human health: lessons from vitamin $D$ receptor null mice. Endocr Rev 2008;29:726-76.

20. Napoli N, Vattikuti S, Ma C, et al. High prevalence of low vitamin D and musculoskeletal complaints in women with breas t cancer. Breast J 2010;16:609-16.

21. Rastelli AL, Taylor ME, Gao F, et al. Vitamin D and aromatase inhibitor-induced musculoskeletal symptoms (AIMSS): a phase II, double-blind, placebo-controlled, randomized trial. Breast Cancer Res Treat 2011;129:107-16.

22. Haussler MR, Whitfield GK, Kaneko I, et al. The role of vitamin D in the FGF23, klotho, and phosphate bone-kidney endocrine axis. Rev Endocr Metab Disord 2012;13:57-69.

23. Lim K, Lu TS, Molostvov G, et al. Vascular Klotho deficiency potentiates the development of human artery calcification and mediates resistance to fibroblast growth factor 23. Circulation 2012;125:2243-55.

24. Wolf I, Levanon-Cohen S, Bose S, et al. Klotho: a tumor suppressor and a modulator of the IGF-1 and FGF pathways in human breast cancer. Oncogene 2008;(56):7094-105.

25. Li YC. Renoprotective effects of vitamin D analogs. Kidney Int 2010;78:134-9.

26. Zhang Z, Zhang Y, Ning G, Deb DK, Kong J, Li YC. Combination therapy with AT1 blocker and vitamin D analog markedly ameliorates diabetic nephropathy: blockade of compensatory renin increase. Proc Natl Acad Sci U S A 2008;105:15896-15901.

27. Oh J, Weng S, Felton SK, et al. 1,25(OH)2 vitamin d inhibits foam cell formation and suppresses macrophage cholesterol uptake in patients with type 2 diabetes mellitus. Circulation 2009;120:687-98.

28. He WC, Kang YS, Dai CS, et al. Blockade of Wnt/beta-catenin signaling by paricalcitol ameliorates proteinuria and kidney injury. J Am Soc Nephrol 2011;22:90-103.

29. de Boer IH, loannou GN, Kestenbaum B, et al. 25-hydroxyvitamin D levels and albuminuria in the Third National Health and Nutrition Examination Survey (NHANES III). Am J Kidney Dis 2007;50:69-77.

30. Chowdhury R, Kunutsor S, Vitezova A, et al. Vitamin D and risk of cause specific death: systematic review and metaanalysis of observational cohort and randomised intervention studies. BMJ 2014;348:g1903.

31. Garland CF, Garland FC, Gorham ED, et al. Meta-analysis of vitamin D sufficiency for improving survival of patients with breast cancer. Anticancer Res 2014;34:1163-6.

32. Dev R, Del Fabbro E, Schwartz GG, et al. Preliminary report: vitamin $\mathrm{D}$ deficiency in advanced cancer patients with symptoms of fatigue or anorexia. Oncologist 2011;16:1637-41.

33. Stone CA, Kenny RA, Healy M, et al. Vitamin D depletion: of clinical significance in advanced cancer? Support Care Cancer 2011;19:865-7.

34. Garland CF, Garland FC. Do sunlight and vitamin D reduce the likelihood of colon cancer? Int J Epidemiol 1980;9:227-31.

35. Garland CF, Garland FC, Gorham ED, et al. The role of vitamin D in cancer prevention. Am J Public Health 2006;96:252-61.

36. EFSA Panel on Dietetic Products, Nutrition and Allergies (NDA). Scientific opinion on the tolerable upper intake level of vitamin D. EFSA J 2012;10:2813. [45 pp.]

37. Groenvold M, Petersen MA, Aaronson NK, et al.; EORTC Quality of Life Group. The development of the EORTC QLQ-C15-PAL: a shortened questionnaire for cancer patients in palliative care. Eur $J$ Cancer 2006;42:55-64

38. Yellen SB, Cella DF, Webster K, et al. Measuring fatigue and other anemia-related symptoms with the Functional Assessment of Cancer Therapy (FACT) measurement system. J Pain Symptom Manage 1997;13:63-74.

39. Mahoney FI, Barthel DW. Functional evaluation: the Barthel index. Md State Med J 1965;14:61-5.

40. Karnofsky DA, Burchenal JH. The clinical evaluation of chemotherapeutic agents in cancer. MacLeod CM. ed. Evaluation of chemotherapeutic agents. Columbia Univ Press, 1949:196.

41. Anderson F, Downing GM, Hill J, et al., Palliative Performance Scale (PPS): a new tool. J Palliat Care 1996;21:5-11.

42. R Core Team. R: A language and environment for statistical computing. Vienna, Austria: R Foundation for Statistical Computing, 2013. http://www.R-project.org/ 